Open Access Article

The authors, the publisher, and the right holders grant the right to use, reproduce, and dis-

seminate the work in digital form to all users.
Journal of Biomolecular Structure \&

Dynamics, ISSN 0739-1102

Volume 23, Issue Number 4, (2006)

CAdenine Press (2006)

\section{Conformational Flexibility of the Peptide Hormone Ghrelin in Solution and Lipid Membrane Bound: A Molecular Dynamics Study}

\author{
http://www.jbsdonline.com
}

\begin{abstract}
Human ghrelin is a peptide hormone of 28 aminoacid residues, in which the Ser3 is modified by an octanoyl group. Ghrelin has a major role in the energy metabolism of the human body stimulating growth hormone release as well as food intake. Here we perform molecular dynamics simulations in explicit water and in a DMPC-lipid bilayer/water system in order to structurally characterize this highly flexible peptide and its lipid binding properties. We find a loop structure with residues Glu17 to Lys 20 in the bending region and a short $\alpha$-helix from residues Pro7 to Glu13. The presence of a lipid membrane does not influence these structural features, but reduces the overall flexibility of the molecule as revealed by reduced root mean square fluctuations of the atom coordinates. The octanoyl-side chain does not insert into the lipid membrane but points into the water phase. The peptide binds to the lipid membrane with its bending region involving residues Arg15, Lys16, Glu17, and Ser18. The implications of these results for the binding pocket of the ghrelin receptor are discussed.
\end{abstract}

Keywords: Ghrelin; Molecular dynamics; Structure; Lipid bilayer; and Receptor binding.

\section{Materials and Methods}

The initial structure of ghrelin was build with Biomer V1.0 (1) in the extended conformation, energy minimized using the built-in Amber forcefield (2) and saved in the PDB file format. All subsequent simulations and trajectory analyses were performed with the GROMACS V3.1.4 software package $(3,4)$. After further in vacuo energy minimisation using the GROMOS96 force field (5), the extended structure was subjected to $200 \mathrm{ps}$ in vacuo MD simulation using a time step of $2 \mathrm{fs}$ at a temperature of $500 \mathrm{~K}$ in order to generate a random ensemble of starting structures for subsequent SAMD simulations in water. The LINCS algorithm was used to constrain all bond-lengths (6). The procedure above was followed in order to obtain an ensemble of randomised starting structures for the subsequent simulations. Structures taken from the in vacuo trajectory at equidistant time points were solvated with SPC water (7) in a periodic truncated octahedron leaving $1.2 \mathrm{~nm}$ of solvent on each side. The ionisation state of the side chains was chosen according to $\mathrm{pH} 7$ with the histidine residue kept uncharged. Five chloride ions were added at positions of favorable electrostatic potential in order to neutralize the charges. After energy minimization the system was subjected to 2000 ps of simulated annealing molecular dynamics (SAMD) changing the temperature continuously from $450 \mathrm{~K}$ to $273 \mathrm{~K}$. The temperature and pressure was set via Berendsen coupling with a time constant of $0.1 \mathrm{ps}$. Electrostatic interactions were treated with a twin-range cut-off method of $2.0 \mathrm{~nm} / 1.5 \mathrm{~nm}$ and Lennard-Jones interactions were calculated with a cut-off radius of $1.5 \mathrm{~nm}$. Other more exact treatments of electrostatic interactions exist, e.g., Ewald mesh methods, which may induce artificial periodicity that may influence peptide conformation (8), but we chose the twin-

\section{Andrew J Beevers Andreas Kukol*}

Department of Biological Sciences

University of Warwick

Coventry CV4 7AL, UK 
range cut-off method to allow for significantly longer simulation times of our

\section{Beevers and Kukol}

Figure 1: The aminoacid sequence of human ghrelin with the octanoyl-group bound to Ser3 as it has been used in all simulations. complex systems. However, we used a higher cut-off radius of $2.0 \mathrm{~nm}$ compared to $1.7 \mathrm{~nm}$ in comparable studies $(9,10)$.

This procedure was performed with 20 starting structures selected from the in vacuo trajectory at equidistant time points. After energy minimization the root mean square deviation (RMSD) between each of the 20 structures was calculated in order to find clusters of similar structures. The water/ions system containing the central structure of a cluster was subjected to a $10 \mathrm{~ns}$ MD simulation at the constant temperature of $298 \mathrm{~K}$ and finally energy minimized. Coordinates of a pre-equilibrated lipid bilayer/water system containing 128 dimyristoyl phosphatidylcholine (DMPC) lipid molecules were obtained from Gurtovenko et al. (11) and the water layer was expanded to a size of 7184 water molecules giving a total size in z-direction (normal to the lipid membrane plane) of $11.9 \mathrm{~nm}$. MD simulations of the lipid bilayer system used the GROMOS-87 forcefield (12) modified with additional forcefield parameters for lipid molecules $(13,14)$. The expanded system was equilibrated by a 1500 ps MD simulation at a temperature of $298 \mathrm{~K}$ and constant atmospheric pressure. The final peptide structure of the $10 \mathrm{~ns}$ water simulation was energy minimized and placed into this lipid bilayer/water system in such orientation that the octanoyl group on Ser3 points to the lipid bilayer. The system was energy minimized and subjected to $100 \mathrm{ps}$ of MD simulations at 298K, while position restraints were applied with a force constant of $1000 \mathrm{~kJ} \mathrm{~mol}^{-1} \mathrm{~nm}^{-2}$ to all peptide atoms. Subsequently two separate unrestrained MD simulations were performed for $15 \mathrm{~ns}$ at $298 \mathrm{~K}$ with different random initial atom velocities chosen from a Maxwell velocity distribution according to the temperature of 298 $\mathrm{K}$. Temperature, pressure coupling, and bond constraints were applied in the same way as for the water simulation described above. Simulations were performed in parallel on an 8-node dual processor LINUX cluster with Intel Xeon $2.66 \mathrm{GHz}$ processors (Streamline Computing, Warwick UK). Images of the structures were made with the VMD molecular viewer (15).

\section{Introduction}

Human ghrelin is a peptide hormone of 28 aminoacid residues, in which the Ser3 is modified by an octanoyl group (Fig. 1). It is the first case of a bioactive peptide, which is modified by a fatty acid that is essential for its activity [reviewed in (16)]. Ghrelin is the natural agonist to a G-protein coupled receptor, which stimulates the release of growth hormone from the pituitary. This receptor, named growth hormone secretagogues receptor (GSHR), because it is stimulated by synthetic compounds, which release growth hormone, is known since 1982, long before the discovery of its natural ligand ghrelin (17). Ghrelin has been found through reverse pharmacology from stomach extract (18) using an orphan receptor strategy (19). Ghrelin is mainly produced in the stomach (20) but also in the kidney (21) and is distributed to several organs through the blood stream. Ghrelin exerts a variety of physiological effects including growth hormone release, appetite stimulation, increasing gastric acid secretion, decrease in blood pressure, and increasing the cardiac index, as well as, stroke volume indices [reviewed in (16)].

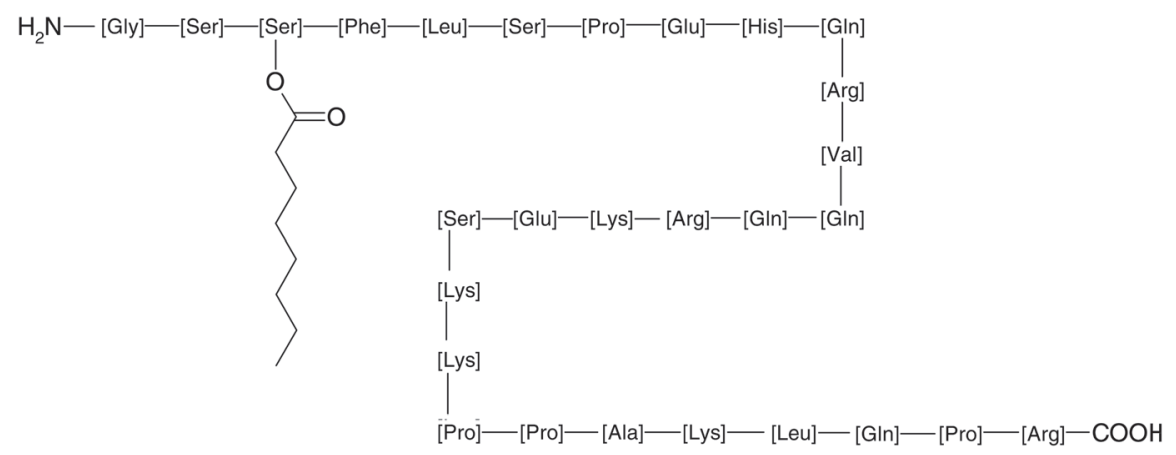


Apart from the primary aminoacid sequence and octanoylation not much is known about the molecular structure of ghrelin. An NMR study shows that ghrelin has no unique structure in solution but possibly exists in a random coil conformation (22). Most likely a conformationally unrestricted peptide will exist in a variety of different fast interconverting conformations, which cannot be resolved by NMR spectroscopy.

With the increase in computer power the application of molecular dynamics (MD) simulations of increasingly complex systems explicitly representing proteins, water molecules, and lipid bilayers has become increasingly widespread [reviewed in $(23,24)]$. The simulation of the folding of a $\beta$-heptapeptide has been attempted successfully using the GROMOS96 forcefield (25) or the folding of a 36 residue protein (26) during a 1 s MD simulation at constant temperature using the Cornell et al. forcefield (2).

Here we use the approach of combining simulated annealing molecular dynamics (SAMD) simulations with 10 ns MD simulations utilizing the GROMOS96 forcefield at constant temperature in aqueous solution in order to study the highly conformationally flexible ghrelin peptide. We also carry out a $15 \mathrm{~ns}$ MD simulation of ghrelin in a lipid bilayer system. The results reveal that parts of the peptide are conformationally restricted giving rise to a short stretch of $\alpha$-helix. The results of the lipid bilayer simulations show that ghrelin binds to lipid bilayers in an unexpected fashion leaving the octanoyl-chain in the aqueous phase, which has implications for the structure of the binding pocket of GSHR.

\section{Results}

Simulated Annealing MD

After reducing the temperature of the simulation from $450 \mathrm{~K}$ to $273 \mathrm{~K}$ over a period of $2 \mathrm{~ns}$ the 20 different extended starting structures obtained from gasphase MD folded into a more compact structure in solution. However, no clusters of similar structures have been found, which have a backbone RMSD among each other of $0.4 \mathrm{~nm}$ or smaller for all 28 residues. Considering only residues 2 to 10 a cluster of 13 structures has been found with a backbone RMSD cut-off of 0.2 $\mathrm{nm}$. The remaining seven structures had a backbone RMSD of more than $0.2 \mathrm{~nm}$ (typically 0.4 to $1.0 \mathrm{~nm}$ ) to any other of the 20 structures. The representative structure of this cluster, which has the lowest backbone RMSD $(<0.2 \mathrm{~nm})$ to all other cluster members, is shown in Figure 2A. The structure has no defined fold apart from a buldge at residues His 9 to Gln 13 indicating $\alpha$-helix formation and a short loop structure involving residues Ser18-Lys20.

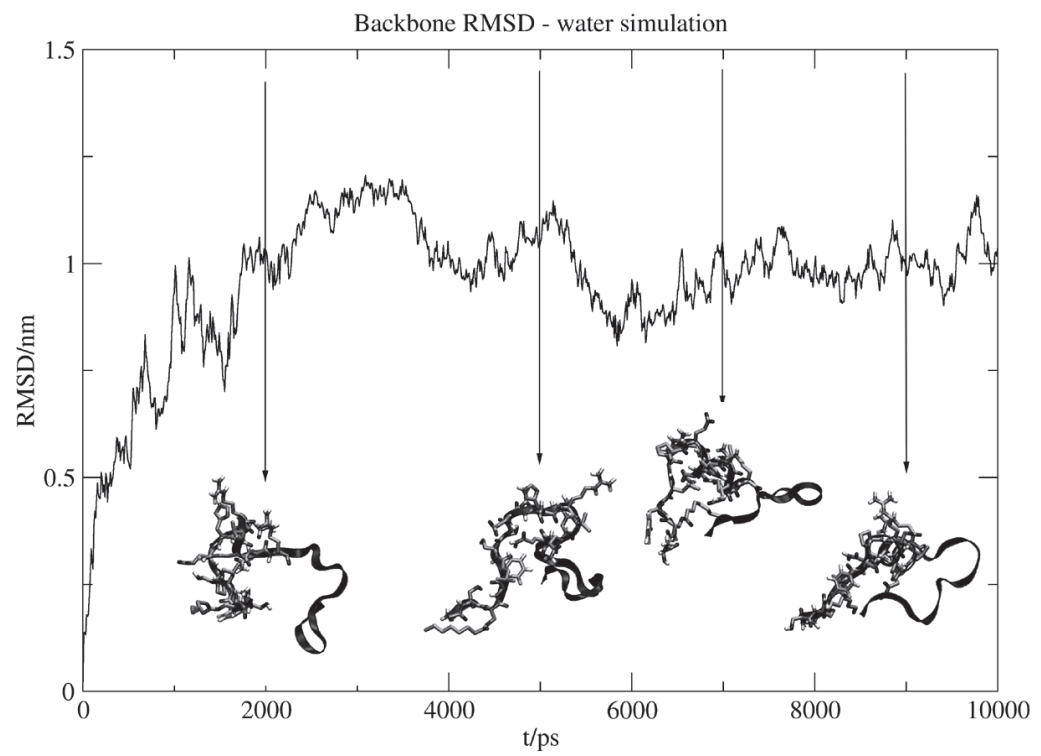

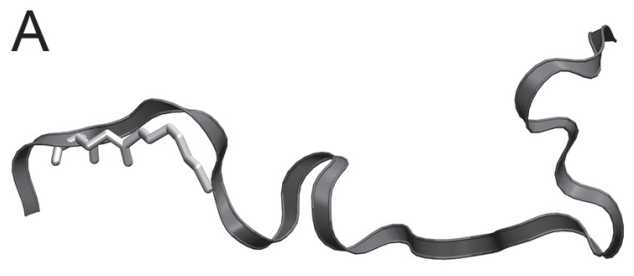

B

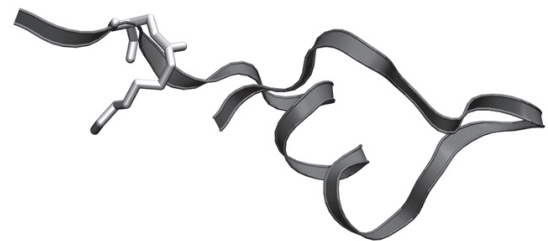

Figure 2: Peptide backbone structures of ghrelin with the octanoyl side chain of Ser3 highlighted. (A) After 2 ns SAMD from $450 \mathrm{~K}$ to $273 \mathrm{~K}$. (B) Structure after $10 \mathrm{~ns}$ MD simulation at $298 \mathrm{~K}$ followed by energy minimization.

Figure 3: Root mean square deviation (RMSD) of the peptide backbone with respect to the starting structure shown in Figure 2A. Snapshots at different time points are shown with residues Gly1 to $\operatorname{Arg} 15$ and the octanoyl-chain highlighted. 


\section{Beevers and Kukol}

Figure 4: Root mean square deviation (RMSD) of the peptide backbone with respect to the starting structure shown in Figure 2B. Snapshots at different time points are shown with the octanoyl-chain highlighted. Only a part of the upper bilayer leaflet is shown. Water molecules are not shown.
Figure 5: The z-coordinates in the direction normal to the lipid bilayer averaged over different groups of atoms, the phosphor atoms in the upper leaflet of the bilayer (black line), the Lys16 backbone atoms (dark grey), and the octanoyl-Ser3 backbone atoms (light grey). The z-coordinates are shown relative the phosphor atoms of the upper lipid bilayer leaflet, which have been set to zero.
The backbone RMSD with respect to the starting structure shown in Figure 3 reveals a significant increase in the first $2.5 \mathrm{~ns}$ of the simulation and then remains around $1 \mathrm{~nm}$ for the rest of the simulation time. This shows that the structure obtained from 2ns of SAMD has not achieved its equilibrium fold. The snapshots taken at different time indicate the structural rearrangements taking place (Fig. 3), i.e., a clear formation of a short $\alpha$-helix from Pro7 to Glu13 and the formation of a hairpin structure with Glu17 to Lys 20 in the bending region. Although these structural elements were already partially indicated in the starting structure obtained from the SAMD only after 2 ns these features become clearly apparent and do not change much during the simulation up to $10 \mathrm{~ns}$. The final energy minimized structure from the water simulation is shown in Figure $2 b$.

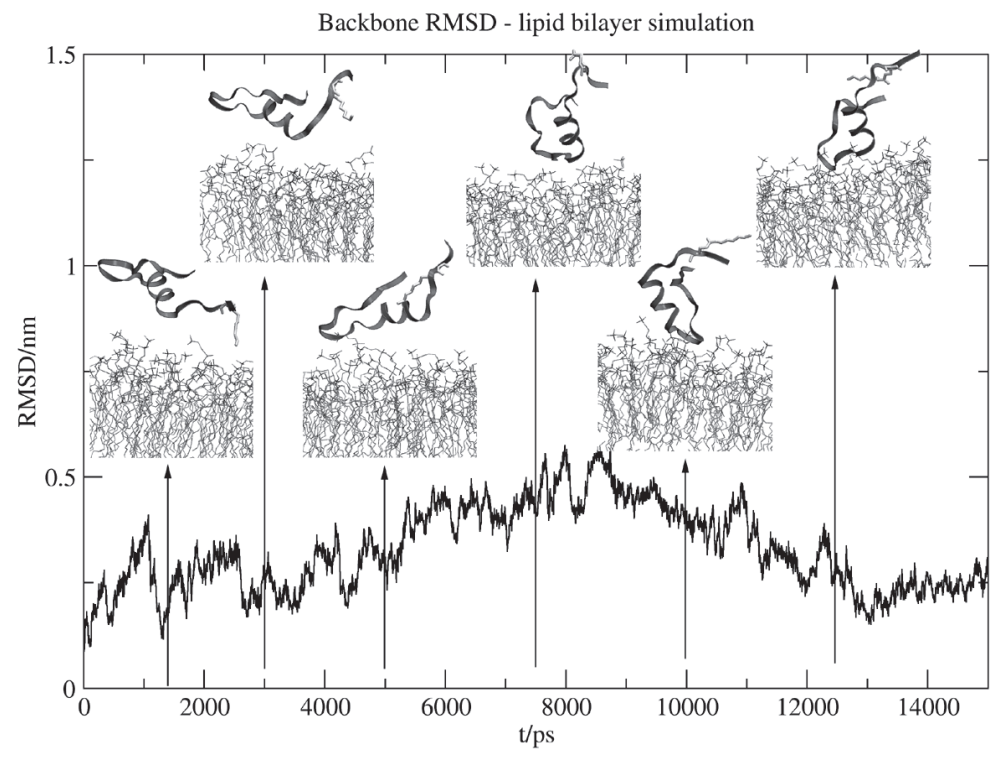

MD Simulation in Water/Lipid Bilayer

The final structure obtained from the $10 \mathrm{~ns}$ water simulation was placed in proximity of the lipid bilayer in such a way that the octanoyl chain on Ser3 was facing the lipid bilayer. This position was chosen to facilitate potential penetration of the octanoyl chain into the lipid bilayer. The RMSD trace of the $15 \mathrm{~ns}$ simulation shown in Figure 4 reveals much less deviation from the starting structure than the water simulation indicating that the presence of the lipid bilayer does not influence

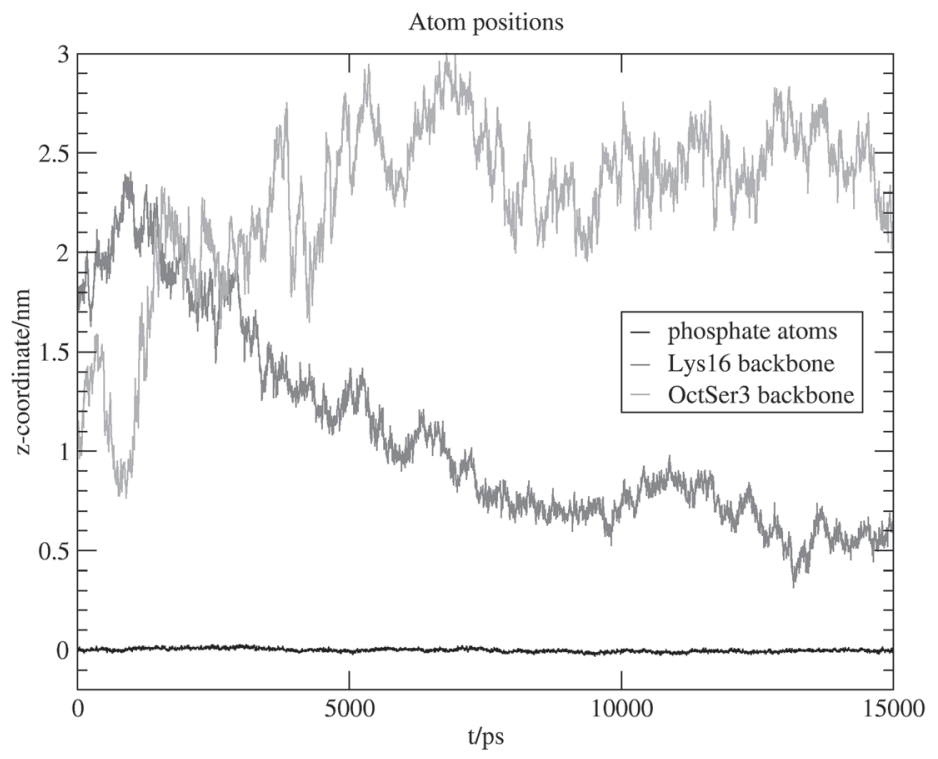




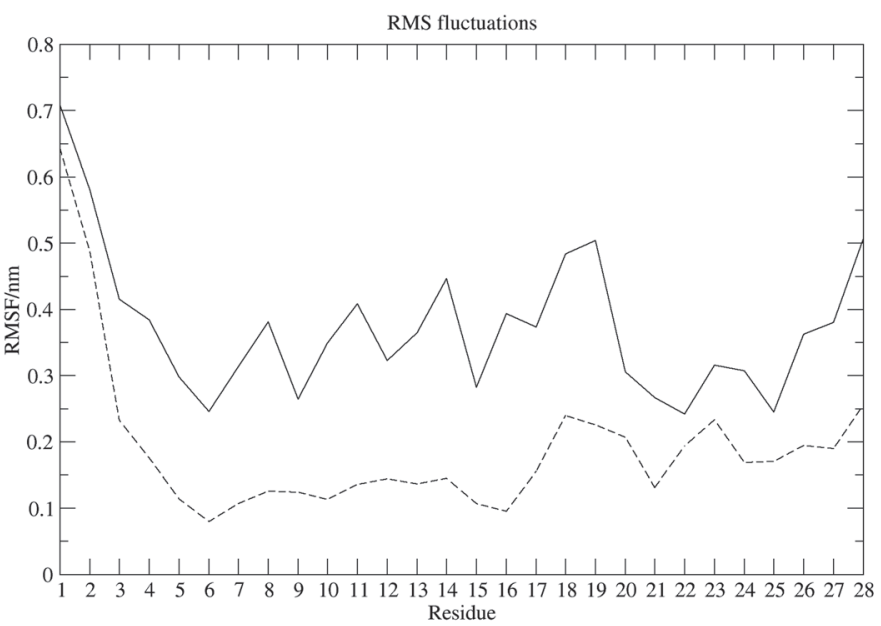

the overall structure. This is also apparent from the visual appearance of the various snapshots shown in Figure 4. As revealed by the snapshots shown in Figure 4 for the first $3 \mathrm{~ns}$ the peptide approaches the lipid bilayer and remains in its initial orientation with respect to the bilayer. From about 5 ns onwards the peptide starts to turn around in the way that the $\mathrm{N}$ - and $\mathrm{C}$-terminus become oriented away from the lipid bilayer towards the water phase, while the loop structure forms a close contact with the lipid bilayer. In particular residues Arg15, Lys16, Glu17, and Ser18 are in close contact with the lipid head groups. Most notably the hydrophobic octanoyl side chain of Ser3 points into the water phase, although it came into close contact with the lipid head groups during the first $2 \mathrm{~ns}$ of the simulation. The residue Lys16 approaches the lipid head group region continuously during the simulation (Fig. 5). While the backbone atoms of Lys16 are at a distance of about $2 \mathrm{~nm}$ to the phosphor atoms of the lipid head groups at the start of the simulation, Lys 16 approaches the phosphor atoms to about $0.6 \mathrm{~nm}$ or close after $8 \mathrm{~ns}$ simulation time. The opposite is true for the octanoylated Ser3 (Fig. 4), which after initially approaching the phosphor atoms up to a distance of $0.8 \mathrm{~nm}$ moves away to a distance of $2.5 \mathrm{~nm}$ or more. These distances represent averages over the three backbone atoms for each residue.

The lipid bilayer reduces the overall conformational flexibility of ghrelin as shown in Figure 6 by the root mean square fluctuations (RMSF) averaged over all atoms for each residue for the last $7.5 \mathrm{~ns}$ of each simulation. Apart from the very flexible $\mathrm{N}$ - and C-termini the lipid bound ghrelin has up to three times lower RMSF values than the solution state ghrelin.

\section{Discussion}

The SAMD simulations did not result in a stable structure as can be seen by comparing Figure $2 \mathrm{~A}$ and B. While the structure in Figure 2B is still highly flexible, it shows some unique structural features (see below), which have not been revealed by the SAMD simulation. Although the time for simulated annealing MD has been increased from 0.75 ns to 2 ns compared to an earlier study for a heptapeptide (25), this time is still too short to find a unique stable structure for a peptide of this size. It can however be used to generate a starting structure for a longer simulation at constant temperature, which may fold into a unique conformation. The formation of a cluster of 13 similar structures obtained from 20 different starting structures is clearly indicative of a unique energy minimum. The structure most similar to all other cluster members had been chosen as a starting point for a subsequent $10 \mathrm{~ns}$ MD simulation at constant temperature. In principle any other cluster member could have been chosen requiring potentially a longer simulation time to adopt a stable conformation. However, any structure outside the cluster would not be able to escape a local energy minimum during a constant temperature simulation at $298 \mathrm{~K}$.

\section{Ghrelin Binding to Lipid Membrane}

Figure 6: The root mean square fluctuations of backbone atoms averaged for each residue for the last $7.5 \mathrm{~ns}$ of the water simulation (solid line) and for the last 7.5 ns of the lipid bilayer/water simulation (dashed line). 
Our results from a $10 \mathrm{~ns}$ MD simulation at constant temperature following a $2 \mathrm{~ns}$ SAMD simulation show that ghrelin has some defined structural features in water solution at $\mathrm{pH} 7$, which consist of a short stretch of $\alpha$-helix from Pro7 to Glu13 and a backwards folding loop with residues Glu17 to Lys 20 in the loop region. It is interesting to compare these results with an ${ }^{1} \mathrm{H}-\mathrm{NMR}$ study (22), which did conclude the absence of any secondary structure features based on the lack on NOE connectivities between hydrogens in successive residues or in residues further apart. The differences may be explained by the $\mathrm{pH}$ of 1 to $1.5 \mathrm{used}$ in the NMR study, which would protonate the two Glu side chains at Glu13 and Glu17, thus introduce two further positive charges to an overall charge of +7 . While ghrelin is produced in the stomach at low $\mathrm{pH}$, its target receptors in various organs are most likely in a solution of $\mathrm{pH} 7$ or above. Based on our simulation results we would not expect the NMR detection of other structural features apart from the short helical region, because of the flexibility of most other parts of the peptide.

Most biological relevant is the behavior of ghrelin in the presence of a lipid bilayer as these may resemble the situation, when ghrelin approaches its receptor. The overall structural features, i.e., the backwards loop of the peptide backbone and the short stretch of $\alpha$-helix, do not change significantly over the whole $15 \mathrm{~ns}$ simulation, which indicates that with regards to these features a stable conformation has been found by SAMD followed by constant temperature MD. The structural features of the octanoyl-serine residue in ghrelin are somewhat reminiscent of a detergent with a hydrophilic head group and a hydrophobic tail. One would expect the hydrophobic octanoyl group to act as an anchor to attach ghrelin to the lipid membrane and in that way facilitate binding of ghrelin to the transmembrane ghrelin receptor. However, our simulations show that this is not the case for our system setup, but ghrelin interacts through positively charged residues with the lipid membrane, while the octanoyl side chain points into the water phase. The same behavior has been observed in a second simulation with different random initial atom velocities (data not shown). We did not attempt to vary the initial placement of ghrelin, because the simulation revealed a complete reorientation of the molecule indicating that all orientations of ghrelin with respect to the lipid bilayer have been sampled. The binding to natural cell membranes would be even stronger, as those have a significant proportion of negatively charged lipids. Our observation has implications for the unknown ghrelin binding site of the receptor. Based on our results we postulate that it consists of a hydrophobic pocket of a size to accommodate the octanoyl chain as well as the hydrophobic side chains of Phe and Leu following the octanoyl-serine in sequence (Fig. 1). These postulates are in line with observations about the minimum structural features of ghrelin required to elicit receptor response (27). It has been shown that a large hydrophobic group is necessary to activate the receptor; full length ghrelin without the octanoyl group is inactive. Furthermore, the first five residues including the octanoyl group are sufficient for ghrelin function (27). If the octanoyl side-chain would act as a mere lipid anchor, ghrelin would still be able to elicit receptor response albeit at higher concentration. The results of our study give the structural basis for the experimental findings mentioned above.

An inherent limitation of all MD simulations is the limited simulation time reasonably available especially for complex systems like a lipid bilayer and its dependence on the starting conditions. During our $15 \mathrm{~ns}$ simulation of the lipid bilayer/ghrelin system the ghrelin peptide adopted a completely different orientation with respect to the lipid bilayer than the starting structure, which is evidence that $15 \mathrm{~ns}$ were long enough to sample all possible orientations and that final orientations were not dependent on the starting structure. Furthermore, the structural features, which have emerged during the $10 \mathrm{~ns}$ water simulation, remain unchanged during the $15 \mathrm{~ns}$ simulation in a different system indicating that these features are a unique property of ghrelin in solution and not an artifact of the simulation or an intermediate stage in folding. 
We have shown that 2 ns SAMD followed by $10 \mathrm{~ns}$ MD at constant temperature is able to yield a structure for a 28 residue peptide, which is highly flexible but shows some stable structural features, which remain during long term simulation even in a different system setup. MD simulations may be one of the few methods available to structurally characterise short peptides, which show a multitude of interconverting conformations.

Our results for the peptide hormone ghrelin show that the octanoyl side-chain does not function as a lipid anchor, but is an essential part for binding to the ghrelin receptor. This will help to identify the binding pocket of the ghrelin receptor as well as defining pharmacologically important analogs and inhibitors of ghrelin function, which may be helpful in treating obesity and diabetes related complications.

\section{Acknowledgements}

This work has been funded by the Biotechnology and Biological Sciences Research Council, UK, grant no. 88/B19450.

\section{References and Footnotes}

1. N. White. http://www.scripps.edu/mb/case/Biomer/ (1999).

2. W. D. Cornell et al. J. Am. Chem. Soc. 117, 5179 (1995).

3. H. J. C. Berendsen, D. van der Spoel, R. van Drunen. Comp. Phys. Chem. Comm. 91 , 43 (1995).

4. E. Lindahl, B. Hess, D. Van der Spoel. J. Mol. Mod. 7, 306 (2001).

5. W. F. van Gunsteren, X. Daura, A. E. Mark. Encyclopedia Comput. Chem. 2, 1211 (1999).

6. B. Hess, H. Bekker, J. G. E. M. Fraaije, H. J. C. Berendsen. J. Comp. Chem. 18, 1463 (1997).

7. H. J. C. Berendsen, J. R. Grigera, T. P. Straatsma. J. Phys. Chem 91, 6269 (1987).

8. W. Weber, P. H. Hueneberger, J. A. McCammon. J. Phys. Chem. B 104, 3668 (2000).

9. R. J. Law, D. P. Tieleman, M. S. Sansom. Biophys. J. 84, 14 (2003).

10. I. H. Shrivastava, D. P. Tieleman, P. C. Biggin, M. S. Sansom. Biophys. J. 83, 633 (2002).

11. A. A. Gurtovenko, M. Patra, M. Karttunen, I. Vattulainen. Biophys. J. 86, 3461 (2004).

12. W. F. Van Gunsteren, H. J. C. Berendsen. Gromos-87 Manual (1987).

13. D. P. Tieleman, H. J. C. Berendsen. Journal of Chemical Physics 105, 4871 (1996).

14. O. Berger, O. Edholm, F. Jahnig. Biophysical Journal 72, 2002 (1997).

15. W. Humphrey, A. Dalke, K. Schulten. J. Mol. Graphics 14, 33 (1996).

16. M. Kojima, K. Kangawa. Physiological Reviews 85, 495 (2005).

17. R. Guillemin et al. Science 218, 585 (1982).

18. M. Kojima et al. Nature 402, 656 (1999).

19. O. Civelli. FEBS Lett. 430, 55 (1998).

20. H. Ariyasu et al. J. Clin. Endocrinol. Metab. 86, 4753 (2001).

21. K. Mori et al. FEBS Lett. 486, 213 (2000).

22. M. V. Silva Elipe, M. A. Bednarek, Y. D. Gao. Biopolymers 59, 489 (2001).

23. M. S. Sansom, D. P. Tieleman, L. R. Forrest, H. J. Berendsen. Biochem. Soc. Trans. 26, 438 (1998).

24. D. P. Tieleman, P. C. Biggin, G. R. Smith, M. S. Sansom. Q. Rev. Biophys. 34, 473 (2001).

25. X. Daura, B. Jaun, D. Seebach, W. F. Van Gunsteren, A. E. Mark. Journal of Molecular Biology 280, 925 (1998).

26. Y. Duan, P. A. Kollman. Science 282, 740 (1998).

27. M. A. Bednarek et al. J. Med. Chem. 43, 4370 (2000).

Date Received: November 8, 2005

Communicated by the Editor Ramaswamy H Sarma 
